\title{
Neutrino electromagnetic properties and neutrino oscillations
}

\section{Carlo Giunti}

INFN, Sezione di Torino, 10125 Torino, Italy

\section{Konstantin Kouzakov*}

Department of Nuclear Physics and Quantum Theory of Collisions, Faculty of Physics, Lomonosov Moscow State University, Moscow 119991, Russia

E-mail: kouzakovegmail.com

\section{Yu-Feng Li}

Institute of High Energy Physics, Chinese Academy of Sciences, Beijing, China

\author{
Alexey Lokhov \\ Institute for Nuclear Research, Russian Academy of Sciences, 117312 Moscow, Russia
}

\author{
Alexander Studenikin \\ Department of Theoretical Physics, Faculty of Physics, Lomonosov Moscow State University, \\ Moscow 119991, Russia \\ Joint Institute for Nuclear Research, Dubna 141980, Moscow Region, Russia
}

\section{Shun Zhou}

Institute of High Energy Physics, Chinese Academy of Sciences, Beijing, China

\begin{abstract}
Magnetic moments of massive neutrinos are brought into focus in the context of neutrino oscillation phenomena. A general framework for the treatment of neutrino spin-flavor oscillations in a magnetic field is presented. Spin and flavor oscillation probabilities are derived in some limiting cases. Possible influences of neutrino magnetic moments on fluxes of Majorana neutrinos from supernovae are discussed.
\end{abstract}

The European Physical Society Conference on High Energy Physics

5-12 July, 2017

Venice

${ }^{*}$ Speaker. 


\section{Introduction}

One of the interesting consequences of nonzero values of neutrino masses is that neutrinos can have electromagnetic properties [1-3]. Among the electromagnetic properties of massive neutrinos the most studied and understood are anomalous magnetic moments [4,5]. The effects of neutrino magnetic moments are searched both in astrophysics and in laboratory measurements. In particular, one might expect manifestations of these effects in astrophysical objects such as magnetars, neutron stars, supernovae, etc., where neutrinos propagate over long distances in the presence of strong magnetic fields. The coupling of neutrino magnetic moments to an external magnetic field can give rise to the phenomenon of neutrino spin-flavor oscillations [6-18] and, hence, can influence the neutrino fluxes from such astrophysical sources.

In the following we formulate an effective equation for treating neutrino spin-flavor oscillations in a magnetic field and outline the potential impact of neutrino magnetic moments on oscillations of Majorana neutrinos from supernovae.

\section{Neutrino oscillations in a magnetic field}

The Hamiltonian of the neutrino magnetic moment interaction with an electromagnetic field is given by

$$
H_{E M}=\frac{1}{2} \sum_{j, k} \mu_{j k} \bar{v}_{j} \sigma_{\mu v} v_{k} F^{\mu v}+\text { h.c. },
$$

where $\mu_{j k}$ are magnetic moments of diagonal $(j=k)$ and transition $(j \neq k)$ types, $F^{\mu v}$ is the electromagnetic field tensor, and $\sigma_{\mu v}=i\left(\gamma^{\mu} \gamma^{v}-\gamma^{v} \gamma^{\mu}\right) / 2$. In the case of a constant uniform magnetic field $\vec{B}$ the Hamiltonian (2.1) acquires the form

$$
H_{E M}=-\sum_{j, k} \mu_{j k} \bar{v}_{j}(\vec{\Sigma} \cdot \vec{B}) v_{k}+h . c ., \quad \vec{\Sigma}=\left(\begin{array}{cc}
\vec{\sigma} & 0 \\
0 & \vec{\sigma}
\end{array}\right),
$$

where $\vec{\sigma}$ is the Pauli matrix.

We limit ourselves to the case of two Dirac neutrino physical states, $v_{1}$ and $v_{2}$, with masses $m_{1}$ and $m_{2}$. For treating neutrino evolution in the presence of a uniform magnetic field in the ultrarelativistic limit, it is customary to employ a four-component basis of the helicity states $v_{1, s= \pm 1}$ and $v_{2, s \pm 1}$. The Schrödinger-like evolution equation is then given by

$$
i \frac{d}{d t}\left(\begin{array}{c}
v_{1, s=1} \\
v_{1, s=-1} \\
v_{2, s=1} \\
v_{2, s=-1}
\end{array}\right)=H_{\text {eff }}\left(\begin{array}{c}
v_{1, s=1} \\
v_{1, s=-1} \\
v_{2, s=1} \\
v_{2, s=-1}
\end{array}\right),
$$

where the effective Hamiltonian $H_{\text {eff }}$ consists of the vacuum and interaction parts,

$$
H_{e f f}=H_{v a c}+H_{E M} .
$$

Taking into account that in the discussed ultrarelativistic limit the chiral and helicity components practically coincide, we transform to the flavor basis using the relations

$$
v_{e}^{R, L}=v_{1, s= \pm 1} \cos \theta+v_{2, s= \pm 1} \sin \theta, \quad v_{\mu}^{R, L}=-v_{1, s= \pm 1} \sin \theta+v_{2, s= \pm 1} \cos \theta
$$


where $v_{e}^{R, L}$ and $v_{\mu}^{R, L}$ are electron and muon neutrino chiral states.

The evolution equation (2.3) in the flavor basis (see, for instance, Ref. [19]) can be recast into a fourth-order homogeneous linear differential equation with constant coefficients, which is exactly solvable. This means that one can derive closed-form expressions for neutrino flavor and spin oscillation probabilities. In a general case these expressions appear to be very cumbersome. However, in some specific situations the oscillation probabilities can be well described with simple formulas. For example, these can be the cases when the neutrino interaction with the longitudinal magnetic-field component $B_{\|}$is negligible and (i) $\omega \gg \mu_{v} B$ or (ii) $\omega \ll \mu_{v} B$, where $\omega=\Delta m_{12}^{2} / 4 E_{v}$ is the vacuum oscillation frequency and $\mu_{v}$ is a putative magnetic moment. In the regime $\omega \gg \mu_{v} B$, assuming that at $t=0$ the neutrino is in the $v_{e}^{L}$ state, the flavor-change and spin-flip probabilities, $P_{v_{e}^{L} \rightarrow v_{\mu}^{L}}$ and $P_{v^{L} \rightarrow v^{R}}$, depend on time according to

$$
P_{v_{e}^{L} \rightarrow v_{\mu}^{L}}(t)=\left[1-P_{v^{L} \rightarrow v^{R}}(t)\right] \sin ^{2} 2 \theta \sin ^{2} \omega t, \quad P_{v^{L} \rightarrow v^{R}}(t)=\sin ^{2} \omega_{B} t,
$$

where $\omega_{B}=\mu_{v} B$. In the regime $\omega \ll \mu_{v} B$, the discussed probabilities are well approximated by

$$
P_{v_{e}^{L} \rightarrow v_{\mu}^{L}}(t)=\cos ^{2} 2 \theta \sin ^{2} \omega_{B} t, \quad P_{v^{L} \rightarrow v^{R}}(t)=\frac{1}{2}(1+\sin 2 \theta) \sin ^{2} 2 \omega_{B} t .
$$

The approximations (2.7) become exact in the limit $\omega / \omega_{B} \rightarrow 0$.

\section{Electromagnetic interactions and oscillations of supernova neutrinos}

A valuable insight into the neutrino magnetic moments can be provided by studies of neutrinos from their intensively energetic sources such as supernovae. It was pointed out long time ago that the neutrino-neutrino refraction in the supernova environment may be very important for neutrino flavor conversions, and recently the nonlinear evolution of neutrino flavors has been found to dramatically change the neutrino energy spectra [20]. Depending on the initial neutrino fluxes and energy spectra, a complete swap between neutrino spectra of electron and non-electron flavors can take place in the whole or a finite energy range, as a direct consequence of collective neutrino oscillations.

The impact of nonzero magnetic moments for massive Majorana neutrinos on collective neutrino oscillations has been explored in Refs. [21,22]. It should be noted that since a Majorana field has half the degrees of freedom of a Dirac field, the electromagnetic properties of massive Majorana neutrinos are reduced such that they can have only transition magnetic moments. For a magnetic field of $10^{12} \mathrm{G}$ and the transition magnetic moment at the level of $10^{-22} \mu_{\mathrm{B}}$, which is just two orders of magnitude larger than the standard-model prediction corresponding to neutrino masses of the order of $0.1 \mathrm{eV}$, the pattern of spectral splits of supernova neutrinos may be observed in future experiments such as the Jiangmen Underground Neutrino Observatory (JUNO) [23, 24]. The identification of the spectral splits will allow probing values of the neutrino magnetic moments which are extremely small and impossible to detect in other terrestrial experiments.

\section{Acknowledgements}

This work was supported by the joint project of the Russian Foundation for Basic Research (RFBR) under grant no. 17-52-53133 GFEN_a and National Natural Science Foundation of China 
(NSFC) under grant no. 1161101153. K.A.K., A.V.L. and A.I.S. also acknowledge support from RFBR under grant no. 16-02-01023 A.

\section{References}

[1] C. Broggini, C. Giunti and A. Studenikin, Adv. High Energy Phys. 2012 (2012) 459526.

[2] C. Giunti and A. Studenikin, Rev. Mod. Phys. 87 (2015) 531.

[3] C. Giunti, K.A. Kouzakov, Y.-F. Li, A.V. Lokhov, A.I. Studenikin and S. Zhou, Ann. Phys. (Berlin) 528 (2016) 198.

[4] B.W. Lee and R.E. Shrock, Phys. Rev. D 16 (1977) 1444.

[5] W.J. Marciano and A.I. Sanda, Phys. Lett. B 67 (1977) 303.

[6] A. Cisneros, Astrophys. Space Sci. 10 (1971) 87.

[7] J. Schechter and J. Valle, Phys. Rev. D 24 (1981) 1883.

[8] L.B. Okun, M.B. Voloshin and M.I. Vysotsky, Sov. Phys. JETP 64 (1986) 446.

[9] E.K. Akhmedov, Sov. J. Nucl. Phys. 48 (1988) 382.

[10] C.-S. Lim and W.J. Marciano, Phys. Rev. D 37 (1988) 1368.

[11] E. Akhmedov and M. Khlopov, Mod. Phys. Lett. A 3 (1988) 451.

[12] J. Vidal and J. Wudka, Phys. Lett. B 249 (1990) 473.

[13] A.Y. Smirnov, Phys. Lett. B 260 (1991) 161.

[14] E.K. Akhmedov, S.T. Petcov and A.Y. Smirnov, Phys. Rev. D 48 (1993) 2167.

[15] G.G. Likhachev and A.I. Studenikin, J. Exp. Theor. Phys. 81 (1995) 419.

[16] M. Dvornikov, J. Phys. G 35 (2008) 025003.

[17] A. Dmitriev, R. Fabbricatore and A. Studenikin, in proceedings of the Corfu Summer Institute 2014, PoS(CORFU2014)050 (2015).

[18] A. Studenikin, EPJ Web Conf. 125 (2016) 04018.

[19] R. Fabbricatore, A. Grigoriev and A. Studenikin, J. Phys.: Conf. Ser. 718 (2016) 062058.

[20] H. Duan, G.M. Fuller and Y.Z. Qian, Ann. Rev. Nucl. Part. Sci. 60 (2010) 569.

[21] A. de Gouvea and S. Shalgar, JCAP 1210 (2012) 027.

[22] A. de Gouvea and S. Shalgar, JCAP 1304 (2013) 018.

[23] F. An et al. [JUNO Collaboration], J. Phys. G 43 (2016) 030401.

[24] J.S. Lu, Y.-F. Li and S. Zhou, Phys. Rev. D 94 (2016) 023006. 